\title{
Конструкции блокирующих слоев для подавления паразитной рекомбинации в мощных диодных лазерах с GaAs волноводом
}

\author{
(C) М.Е. Муретова ${ }^{1}$, Ф.И. Зубов ${ }^{1,2, \uparrow ~, ~ Л . В . ~ А с р я н ~}{ }^{3}$, Ю.М. Шерняков ${ }^{4}$, М.В. Максимов ${ }^{1,2}$, А.Е. Жуков ${ }^{2,1}$ \\ ${ }^{1}$ Санкт-Петербургский национальный исследовательский Академический университет им. Ж.И. Алфёрова \\ Российской академии наук, \\ 194021 Санкт-Петербург, Россия \\ ${ }^{2}$ Национальный исследовательский университет „Высшая школа экономики“, \\ 190008 Санкт-Петербург, Россия \\ ${ }^{3}$ Virginia Polytechnic Institute and State University, \\ Blacksburg, Virginia 24061, USA \\ ${ }^{4}$ Физико-технический институт им. А.Ф. Иоффе Российской академии наук, \\ 194021 Санкт-Петербург, Россия \\ ๑E-mail: fedyazu@mail.ru
}

Поступила в Редакцию 18 октября 2021 г.

В окончательной редакции 15 ноября 2021 г.

Принята к публикации 19 ноября 2021 г.

На основе численного моделирования выполнен поиск асимметричных барьерных слоев (АБС) для лазерного диода с волноводом GaAs, излучающего на длине волны $\lambda=980$ нм. Пара АБС, прилегающих к активной области по обе стороны, блокирует нежелательные потоки носителей заряда и снижает паразитную спонтанную рекомбинацию в волноводных слоях. Предложены оптимальные конструкции АБС на основе $\mathrm{AlGaAsSb}$ и $\mathrm{GaInP}$ для блокирования электронов и дырок соответственно, позволяющие уменьшить ток паразитной рекомбинации до менее чем $1 \%$ от исходного. Для подавления транспорта электронов также предложена альтернативная конструкция на основе трех одинаковых AlInAs-барьеров. Спейсеры GaAsP, разделяющие эти барьеры друг от друга, имеют разную толщину. Вследствие этого в каждом спейсере формируется свой собственный набор квазисвязанных (резонансных) состояний, отличающийся от набора состояний соседнего спейсера, что приводит к перекрытию каналов резонансного туннелирования: паразитный поток электронов снижается в несколько десятков раз в сравнении со случаем использования спейсеров равной толщины.

Ключевые слова: полупроводниковые лазеры, асимметричные барьерные слои, паразитная волноводная рекомбинация, резонансное туннелирование.

DOI: 10.21883/FTP.2022.03.52125.9755

\section{1. Введение}

Паразитная спонтанная рекомбинация в волноводных слоях полупроводниковых лазеров является одной из основных причин, ограничивающих эффективность этих приборов [1]. Хотя паразитная рекомбинация имеет место во всех лазерах традиционных конструкций, наиболее остро она проявляется в коротковолновых приборах (с $\lambda \lesssim 1$ мкм для GaAs-волновода), при использовании широких волноводов (1 мкм и более, что характерно для мощных излучателей), при высоких плотностях тока ( $\gtrsim 10$ кА/ $\mathrm{cm}^{2}$, что типично не только для мощных полосковых лазеров, но и для микродисковых лазеров), а также в случае работы при повышенной температуре, в том числе из-за саморазогрева [2]. Например, в мощных лазерных диодах с $\lambda \approx 1$ мкм с широким (4 мкм) GaAs-волноводом имеет место настолько сильная паразитная рекомбинация, что лазерная генерация становится возможной только в условиях пониженных температур [2]. При этом использование именно GaAs-волновода является желательным ввиду его наибольших теплопроводности и электрической проводимости среди материалов AlGaAs.
Перспективным способом подавления паразитной волноводной рекомбинации является использование специальных блокирующих слоев, так называемых асимметричных барьерных слоев (АБС), прилегающих к активной области по обе стороны (рис. 1) [3]. АБС со стороны $p$-эмиттера ( $n$-эмиттера) формирует в волноводном слое высокий энергетический барьер для электронов (дырок), препятствующий их нежелательному транспорту - попаданию в волноводный слой, прилегающий к $p$-эмиттеру (n-эмиттеру); причем этот же АБС создает для дырок (электронов) незначительный побочный барьер (или неглубокую яму, см. рис. 1), так что они свободно попадают в активную область. В идеальном случае полного блокирования нежелательных потоков волноводные слои оказываются заселены носителями только одного типа, и рекомбинация происходит только в активной области.

Первые лазеры с АБС были продемонстрированы в работах [4,5]. Однако улучшение характеристик за счет использования АБС оказалось менее значительным, нежели то, что теоретически предсказывалось для полного блокирования паразитных потоков [6,7]. Причина 


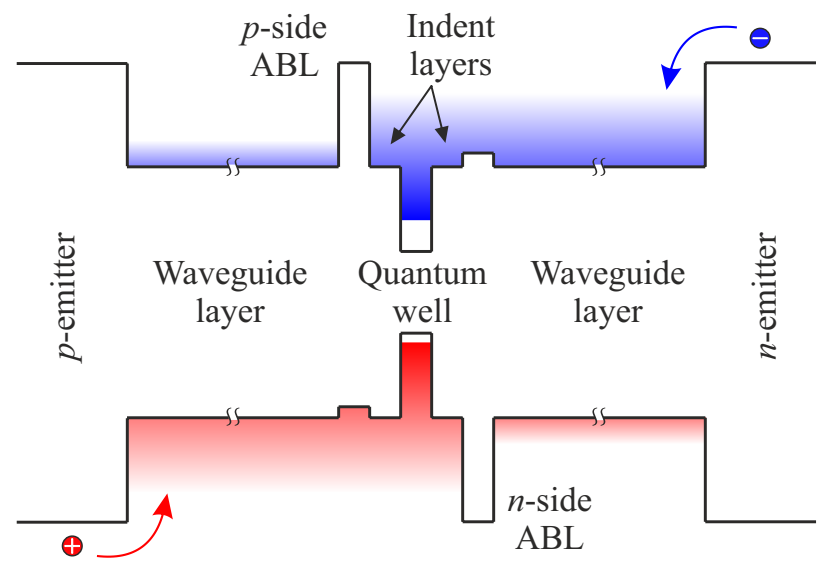

Рис. 1. Схематическая зонная энергетическая диаграмма лазера с асимметричными барьерными слоями (АБС/ABL).

такого несоответствия кроется в том, что степень подавления паразитного потока, определяемая как отношение паразитного потока в отсутствие АБС к потоку при его наличии, для электронов в созданных лазерах была невелика (не более 20). К тому же теоретическая модель не допускала возможных туннельных утечек носителей сквозь АБС, которые неизбежно происходят в создаваемых на практике лазерах с АБС. Недавно нами была развита модель лазеров с АБС, учитывающая туннелирование носителей заряда сквозь АБС [8]. Для лазера с $\lambda=980$ нм и с волноводом GaAs было установлено, что для заметного улучшения приборных характеристик степени подавления нежелательных потоков должны быть $\gtrsim 10^{4}$.

В настоящей работе для лазерной гетероструктуры с волноводом GaAs мы выполняем поиск конструкций АБС, позволяющих достичь требуемых степеней подавления паразитных потоков носителей заряда.

\section{2. Методика расчета}

Поиск осуществлялся среди тройных и четверных соединений $\mathrm{A}^{\mathrm{III}} \mathrm{B}^{\mathrm{V}}$, образуемых элементами (Al, Ga и $\mathrm{In})$ и (As, $\mathrm{P}$ и $\mathrm{Sb})$. Основной целью поиска являлось определение оптимальных конструкций блокирующих слоев, а именно их составов и толщин, позволяющих максимально увеличить коэффициент $C$ подавления паразитных потоков электронов или дырок относительно потоков в структуре без АБС.

Увеличение коэффициента $C$, как правило, сопровождается некоторым подавлением полезного потока носителей за счет появления побочного барьера или ямы, описываемого коэффициентом $S$, определяемого как отношение полезного потока в отсутствии АБС к потоку при его наличии. Ранее нами было показано, что если $C \gtrsim 10^{4}$, то возрастание $S$ вплоть до $\approx 100$ является допустимым с точки зрения эффективного подавления паразитной рекомбинации и не приводит к существенному ухудшению статических характеристик прибора [8]. Это обстоятельство значительно расширяет класс материалов, которые могут быть использованы для создания АБС. В настоящей работе в процессе поиска мы полагали, что максимальная степень подавления полезного потока носителей не должна превосходить $S=100$. Другие использованные ограничения: рассогласование $\delta$ постоянной решетки АБС относительно подложки GaAs не должно превышать 2.5\%; толщина АБС ограничена критической толщиной $h_{\mathrm{c}}$ образования дислокаций, рассчитываемой на основе модели Мэтьюса-Блэксли.

Потоки носителей заряда при наличии и в отсутстие АБС определялись с помощью формулы Тсу-Есаки [9] посредством численного интегрирования, как описано в работе [10]. Вычисляемые потоки сквозь АБС включали как туннельное пропускание носителей заряда с энергиями, не превышающими энергетический барьер, так и надбарьерное прохождение носителей с энергиями больше энергии барьера. Учитывался транспорт носителей, заселяющих Г-, X- и L-долины зоны проводимости, а также подзоны тяжелых дырок, легких дырок и спин-отщепленную подзону валентной зоны. Вероятность туннелирования носителей сквозь барьерные структуры вычислялась по методу матриц переноса. В процессе поиска толщины АБС варьировались с шагом 0.1 нм в диапазоне от 3 нм до толщины, при которой наступает насыщение коэффициента $C$, или до критической толщины $h_{\mathrm{c}}$, если она меньше. Состав всех слоев полагался постоянным на протяжении их толщины. Материальные параметры полупроводниковых соединений брались из работ $[11,12]$. Учитывалось нелинейное поведение материальных параметров в зависимости от состава, а также влияние упругих напряжений на положение краев зон.

Для выбранных конструкций АБС осуществлялся расчет плотности остаточного паразитного тока рекомбинации в волноводных слоях $j_{\text {res }}$ модельного полоскового лазера $(\lambda=980$ нм). В нем квантовая яма (КЯ) $\mathrm{Ga}_{0.82} \mathrm{In}_{0.18} \mathrm{As}$ толщиной 7.6 нм расположена в середине волновода GaAs толщиной 800 нм, заключенного между обкладками $\mathrm{Al}_{0.2} \mathrm{Ga}_{0.8} \mathrm{As.}$ Считалось, что АБС прилегают к активной области вплотную. Длина полоска полагалась равной 500 мкм, а ширина - 3 мкм. Также полагалось, что одно зеркало глухое, а другое - без напыления. Ток паразитной рекомбинации вычислялся в предположении нейтральной КЯ с использованием модели, предложенной в работе [8]. Температура гетероструктур полагалась равной $300 \mathrm{~K}$.

\section{3. Результаты и обсуждение}

На рис. 2 представлены расчетные значения степени подавления паразитных потоков электронов $\left(C_{\mathrm{e}}\right)$ для АБС на основе соединений AlGaInAs (рис. 2, $a$ ) и $\mathrm{AlGaAsSb}$ (рис. 2,b) и дырок $\left(C_{\mathrm{h}}\right)$ для АБС GaInP 

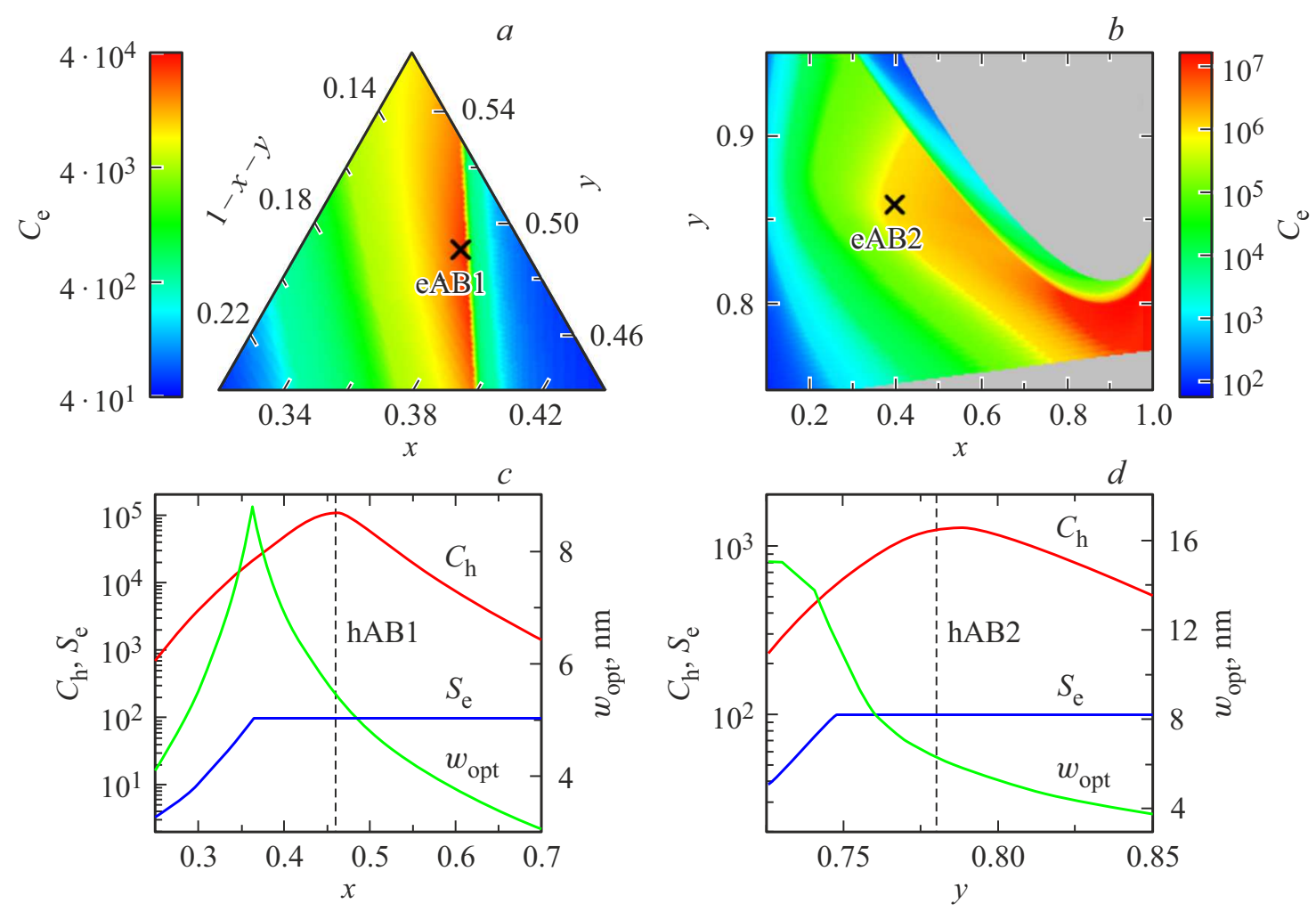

Рис. 2. Степень подавления паразитных потоков электронов $C_{\mathrm{e}}$ для АБС на основе $\mathrm{Al}_{x} \mathrm{Ga}_{y} \mathrm{In}_{1-x-y} \mathrm{As}_{(a)}$ и $\mathrm{Al}_{x} \mathrm{Ga}_{1-x} \mathrm{As}_{y} \mathrm{Sb}_{1-y}(b)$ в зависимости от состава соединений. Степени подавления паразитных потоков дырок $C_{\mathrm{h}}$, полезных потоков электронов $S_{\mathrm{e}}$ и

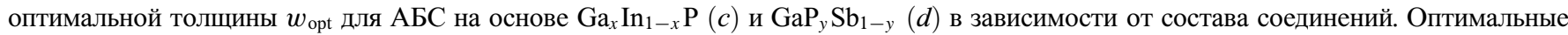
составы обозначены как еАB1, eAB2, hAB1 и hАB2. (Цветной вариант рисунка представлен в электронной версии статьи).

Таблица 1. Основные параметры оптимальных конструкций АБС для блокирования паразитного потока электронов (еАВ1 и еАВ2) и блокирования паразитного потока дырок (hAB1 и hAB2)

\begin{tabular}{c|c|c|c|c}
\hline \multirow{2}{*}{ Соединение } & eAB1 & eAB2 & hAB1 & hAB2 \\
\cline { 2 - 5 } & $\mathrm{Al}_{0.37} \mathrm{Ga}_{0.49} \mathrm{In}_{0.14} \mathrm{As}$ & $\mathrm{Al}_{0.4} \mathrm{Ga}_{0.6} \mathrm{As}_{0.86} \mathrm{Sb}_{0.14}$ & $\mathrm{Ga}_{0.46} \mathrm{In}_{0.54} \mathrm{P}$ & $\mathrm{GaP}_{0.78} \mathrm{Sb}_{0.22}$ \\
\hline$C$ & $3.3 \cdot 10^{4}$ & $1.3 \cdot 10^{6}$ & $1.0 \cdot 10^{5}$ & $1.2 \cdot 10^{3}$ \\
$S$ & 91 & 3 & 95 & 99 \\
$\delta, \%$ & -1.04 & -1.17 & -0.42 & 1.09 \\
$w_{\text {opt}, \mathrm{HM}}$ & 9.8 & 8.4 & 5.4 & 6.3 \\
$h_{\mathrm{c}}, \mathrm{Hм}$ & 9.8 & 8.4 & 30.8 & 9.2
\end{tabular}

Примечание. $C(S)$ - степень подавления паразитного (полезного) потока, $\delta$ - рассогласование по постоянной решетки относительно GaAs, $w_{\text {opt }}$ - оптимальная толщина, $h_{\mathrm{c}}$ - критическая толщина образования дислокаций.

(рис. 2,c) и GaPSb (рис. 2, d) в зависимости от состава. В результате поиска были найдены следующие оптимальные конструкции АБС, обеспечивающие наибольшие значения $C_{\mathrm{e}}$ или $C_{\mathrm{h}}$ : для подавления транспорта электронов - $9.8 \mathrm{Hм} \mathrm{Al}_{0.37} \mathrm{Ga}_{0.49} \mathrm{In}_{0.14} \mathrm{As}$ и $8.4 \mathrm{Hм}$ $\mathrm{Al}_{0.4} \mathrm{Ga}_{0.6} \mathrm{As}_{0.86} \mathrm{Sb}_{0.14}$ (обозначенные нами еАB1 и еАВ2 соответственно), а для подавления транспорта дырок5.4 нм Ga $\mathrm{Ga}_{0.46} \mathrm{In}_{0.54} \mathrm{P}$ и 6.3 нм $\mathrm{GaP}_{0.78} \mathrm{Sb}_{0.22}$ (обозначенные нами hAB1 и hАB2 соответственно). На панелях $c$ и $d$ рис. 2 также приведены зависимости степени подавления полезного потока электронов $S_{\mathrm{e}}$, а также оптимальной толщины $w_{\text {opt }}$ от состава для АБС на основе соединений
GaInP и GaPSb соответственно. В табл. 1 представлены основные параметры выбранных АБС. Конструкция eAB1 на основе соединения AlGaInAs дает степень подавления потока электронов $C_{\mathrm{e}}=3.3 \cdot 10^{4}$. АБС еАВ2, содержащий сурьму, позволяет существенно (в 39 раз) повысить $C_{\text {e }}$ до $1.3 \cdot 10^{6}$. Толщина АБС в случае еАВ1 и еАВ2 лимитирована критической толщиной образования дислокаций $h_{\mathrm{c}}$. Коэффициент подавления полезных потоков дырок составляет в этих структурах $S_{\mathrm{h}}=91$ и 3 соответственно.

Очень высокую степень подавления паразитного потока дырок $C_{\mathrm{h}}=1.0 \cdot 10^{5}$ дает конструкция $\mathrm{hAB} 1$ на 
Таблица 2. Остаточный ток паразитной рекомбинации в волноводе лазера для различных комбинаций конструкций АБС по отношению к случаю лазера без АБС, \%

\begin{tabular}{c|r|r|r}
\hline & eAB1 & eAB2 & TAB \\
\hline hAB1 & 6.3 & 0.3 & 1.0 \\
hAB2 & 17.5 & 12.2 & 12.7
\end{tabular}

основе простого тройного твердого раствора GaInP. Альтернативный дизайн hAB2, содержащий сурьму, имеет степень подавления на 2 порядка ниже $-C_{\mathrm{h}}=1.2 \cdot 10^{3}$. Этот АБС имеет смысл использовать в том случае, когда требуется внедрить слой с положительным рассогласованием (т.е. решетка растянута) для компенсации напряжений, вносимых другими слоями с $\delta<0$. В обоих случаях hАВ1 и hАВ2 оптимальная толщина АБС определяется условием $S \leq 100$.

В табл. 2 представлен остаточный паразитный ток волноводной рекомбинации $j_{\text {res }}$ для различных комбинаций электронных и дырочных АБС по отношению к паразитному току в лазере без АБС. Плотность тока накачки лазера полагалась 5.0 кА/см². Плотность тока паразитной волноводной рекомбинации в отсутствие АБС составляла $j_{\mathrm{par}}^{\mathrm{ref}}=2.0 \kappa \mathrm{A} / \mathrm{cm}^{2}$. Видно, что при использовании комбинации hAB1 (GaInP) и еAB2 (AlGaAsSb) практически полностью удается устранить паразитную волноводную рекомбинацию $\left(j_{\text {res }}\right.$ составляет $<1 \%$ от $j_{\text {par }}^{\text {ref }}$. Подавление этого канала рекомбинации в свою очередь ведет к тому, что накачка практически полностью расходуется на полезную рекомбинацию в активной области лазера, что приводит к соответствующему увеличению излучаемой мощности. Для комбинации hAB1 (GaInP) и eAB1 (AlGaInAs) остаточный ток несколько выше - 6.3\% от исходного. Использование hAB2 (GaPSb) является еще менее эффективным: $j_{\text {res }}$ составляет 17.5 и $12.2 \%$ от $j$ par для комбинаций с еАB1 и еАВ2 соответственно. Также в табл. 2 приведены результаты расчетов для альтернативной конструкции ТАВ, которая обсуждается далее.

На рис. 3 представлена энергетическая диаграмма барьеров, формируемых предложенными АБС в $\mathrm{GaAs}$ для Г- и L-долин зоны проводимости, а также hh- и lh-подзон валентной зоны. АБС еАВ2 формирует для Г- и L-долин высокие барьеры 464 и 131 мэВ, тогда как еАВ1 для них создает барьеры существенно ниже - 267 и 45 мэВ соответственно. Из-за этого еАВ1 значительно хуже справляется с блокированием Г- и L- электронов, чем eAB2. Для еAB1 остаточные потоки Г- и L-электронов составляют 1.8 и $2.1 \mathrm{~A} / \mathrm{cm}^{2}$, а для еАВ2 - $1.4 \cdot 10^{-2}$ и $5.4 \cdot 10^{-2} \mathrm{~A} / \mathrm{cm}^{2}$ соответственно.

Хотя АБС hАВ1 формирует сравнительно невысокий барьер для дырок (281 мэВ), его вполне хватает, чтобы обеспечить хорошее подавление паразитного потока дырок, так как туннелирование частиц с большей массой происходит с меньшей вероятностью. Добавим, что

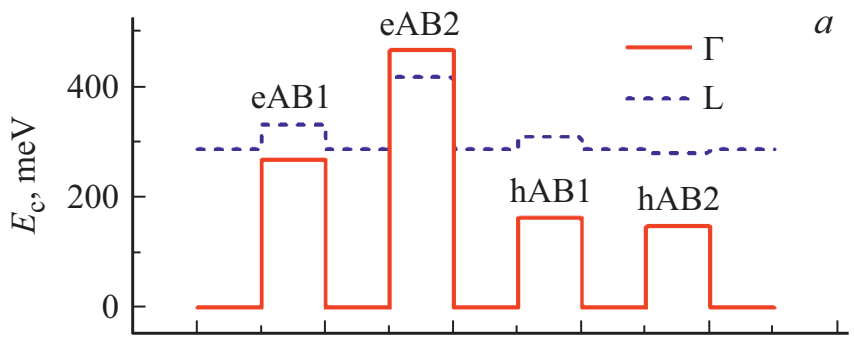

Growth direction, [001]

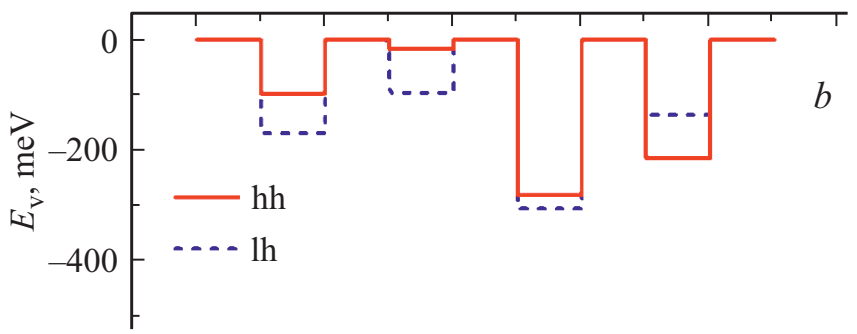

Рис. 3. Энергетические диаграммы барьеров, формируемых оптимальными конструкциями АБС в GaAs для Г- и $\mathrm{L}$-долин $(a)$, а также для hh- и lh-подзон $(b)$.

вклад Х-долин и so-подзоны в потоки носителей оказался пренебрежимо мал из-за очень большой энергии отщепления их от дна зоны проводимости и потолка валентной зоны в $\mathrm{GaAs}$ (477 и 341 мэВ соответственно).

В процессе поиска АБС с использованием приведенных выше ограничений значительная группа соединений, формирующих высокие барьеры для электронов, была отвергнута. Это связано с тем, что они имеют большое рассогласование $\delta$ и, соответственно, малую критическую толщину $h_{\mathrm{c}}$, не позволяющую создать достаточно толстый АБС и достичь высоких значений $C_{\mathrm{e}}$, т.е. реализовать потенциал высокого барьера. Так, соединение $\mathrm{Al}_{x} \mathrm{In}_{1-x} \mathrm{As} \mathrm{c} x$ в диапазоне от 0.66 до 0.69 формирует в $\mathrm{GaAs}$ барьеры для Г- и L-долин высотой не менее 526 и 176 мэВ соответственно, а побочный барьер для дырок остается умеренным, не более 129 мэВ (рис. 4, $a$ ). Однако для этих соединений рассогласование $|\delta|$ составляет

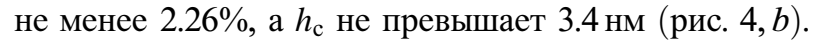

При внедрении в $\mathrm{GaAs}$ АБС $\mathrm{Al}_{0.66} \mathrm{In}_{0.34} \mathrm{As}$ с толщиной, равной его критической толщине (3 нм), достигается степень подавления паразитного потока электронов, равная всего 627. Однако если за этим слоем расположить спейсерный слой, компенсирующий напряжения барьера, но не приводящий к сильному снижению полезного потока, и добавить еще один АБС, идентичный исходному, то степень блокирования паразитного потока можно заметно увеличить. На рис. 5, $a$ показаны зависимости степеней подавления паразитного потока электронов $C_{\mathrm{e}}$ и полезного потока дырок $S_{\mathrm{h}}$ от числа таких барьеров, разделенных спейсерами, в предположении, что энергетические зоны спейсеров такие же, как у GaAs, a их толщина равна 3 нм. Добавление второго барьера в 17 раз увеличивает $C_{\mathrm{e}}$, a $S_{\mathrm{h}}$ остается в пределах допустимого значения $(\leq 100)$. Однако последующее 

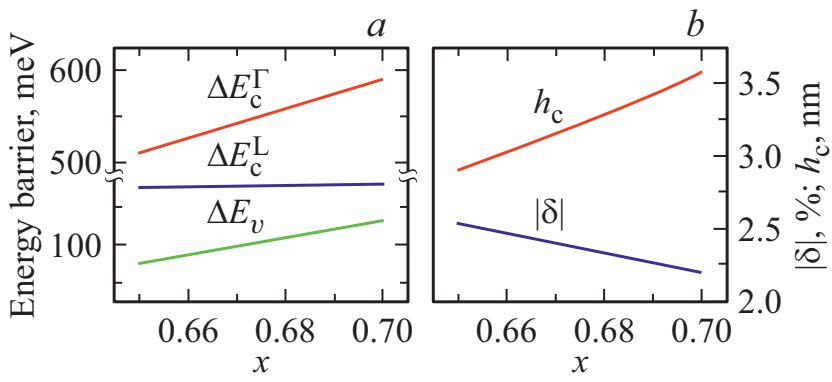

Рис. 4. Энергетические барьеры $\Delta E_{\mathrm{c}}^{\Gamma}$ и $\Delta E_{\mathrm{c}}^{L}$ для электронов $\Gamma$ - и L-долин соответственно и энергетический барьер $\Delta E_{v}$ для дырок, формируемые АБС $\mathrm{Al}_{x} \operatorname{In}_{1-x} \mathrm{As}$ в $\mathrm{GaAs}(a)$, a также рассогласование $|\delta|$ и критическая толщина образования дислокаций $h_{\mathrm{c}}$ для этих соединений $(b)$ в зависимости от мольной доли алюминия $(x)$.

добавление таких АБС практически не меняет поток сквозь структуру. Это связано с тем, что квази-связанные состояния в соседних спейсерах имеют одинаковые энергии, что формирует каналы эффективного резонансного туннелирования носителей. Если же параметры одного из спейсеров, например его толщину, поменять, то поменяется и спектр его квази-связанных состояний (см. вставку на рис. $5, c$ ), из-за чего каналы резонансного туннелирования носителей будут перекрыты и поток сквозь структуру упадет.

На рис. $5, b$ показаны спектры коэффициентов туннелирования (пропускания) электронов сквозь два барьера $\mathrm{Al}_{0.66} \mathrm{In}_{0.34} \mathrm{As}$ толщиной 3 нм, разделенных спейсерами 3,4 и 5 нм. Пики в спектрах соответствуют положениям квазисвязанных состояний спейсеров. Видно, что изменение толщины спейсера всего на 1 нм приводит к существенной перестройке положений квазисвязанных состояний. На рис. 5, с представлены зависимости степеней подавления паразитного потока электронов $C_{\mathrm{e}}$ и полезного потока дырок $S_{\mathrm{h}}$ для структуры из трех 3 нм $\mathrm{AБC} \mathrm{Al}_{0.66} \mathrm{In}_{0.34} \mathrm{As}$, разделенных спейсерами, имеющими такие же зоны, как у GaAs, в зависимости от толщины второго спейсера, при толщине первого, равной 3 нм. Видно, что существует оптимальная толщина второго спейсера, примерно равная 6.3 нм, при которой одновременно достигается близкое к максимальному значение $C_{\mathrm{e}}=2.9 \cdot 10^{5}$ (в 26 раз превышающему $C_{\mathrm{e}}$ для случая, когда спейсеры равны по толщине) и минимальное $S_{\mathrm{h}}=81$.

В результате дополнительного поиска было обнаружено, что функцию спейсеров, компенсирующих напряжения АБC AlInAs, могут выполнять простые тройные соединения $\mathrm{GaAs}_{y} \mathrm{P}_{1-y}$ с $y$ в диапазоне от 0.6 до 0.9. На рис. 6 представлены основные параметры таких спейсеров, внедренных в слой GaAs: энергетические барьеры $\Delta E_{\mathrm{c}}^{\Gamma}, \Delta E_{\mathrm{c}}^{\mathrm{L}}, \Delta E_{v}$, формируемые для $\Gamma$-, L-электронов в зоне проводимости и дырок в валентной зоне (рис. $6, a$ ) соответственно, а также рассогласование $\delta$, критическая толщина $h_{\mathrm{c}}$ и толщина спейсера $w_{\mathrm{sp}}$, требуемая для компенсации напряжений 2.5 нм АБС $\mathrm{Al}_{0.66} \mathrm{In}_{0.34} \mathrm{As}$ в зависимости от мольной доли мышьяка $(y)$. Так, например, для $y=0.8$ барьер для дырок составляет всего 53 мэВ, а компенсация напряжений достигается при толщине 8.7 нм. Мы видим, что изменение состава спейсера $\mathrm{GaAs}_{y} \mathrm{P}_{1-y}$ позволяет менять его толщину, необходимую для релаксации напряжений АБС, и, соответственно, позволяет менять спектр квазисвязанных состояний между барьерами.
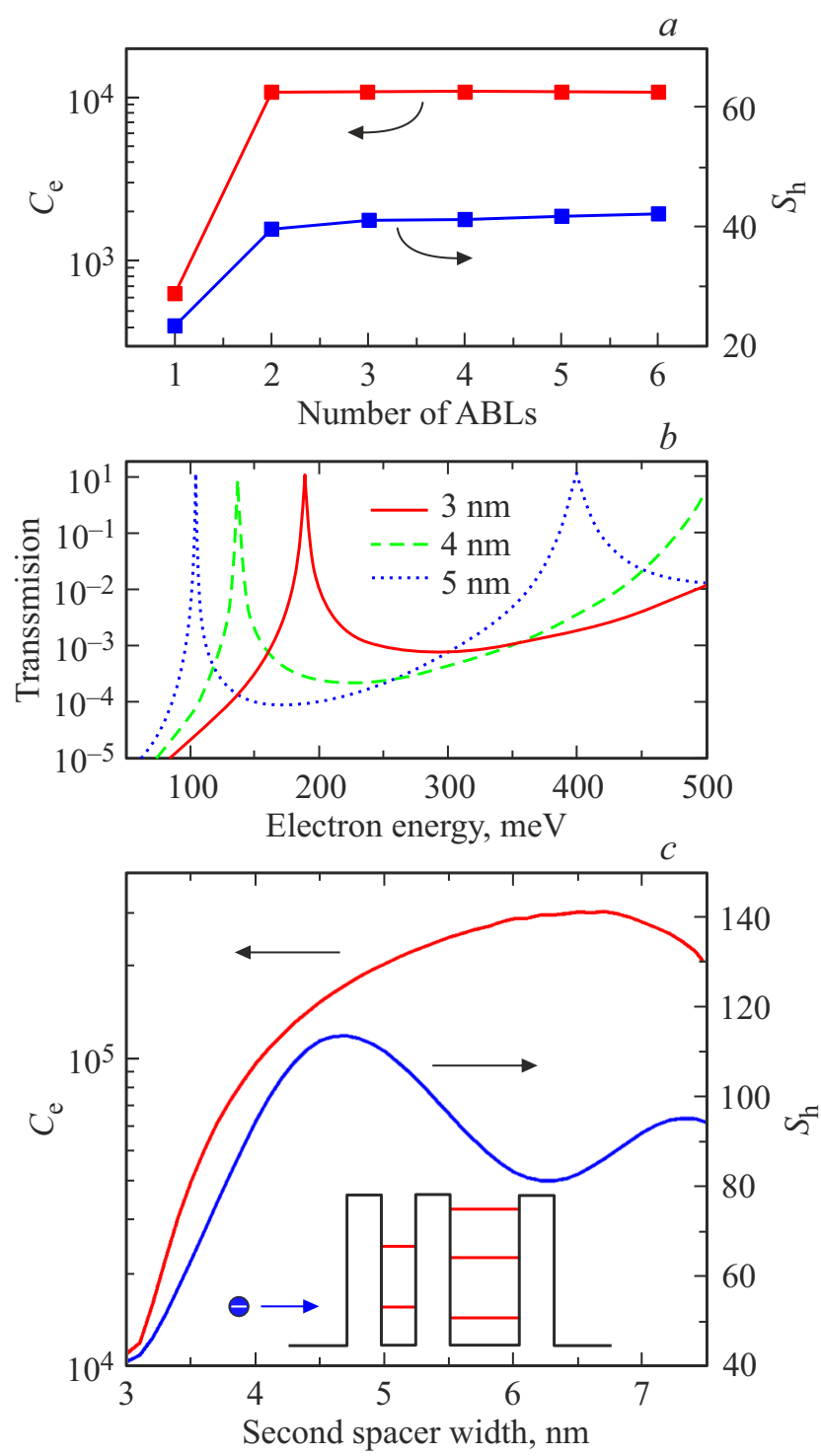

Рис. 5. $a-$ зависимость степени подавления паразитного потока электронов $C_{\mathrm{e}}$ и полезного потока дырок $S_{\mathrm{h}}$ от числа АБС, разделенных спейсерами толщиной 3 нм. $b-$ спектры коэффициента туннелирования (пропускания) электронов сквозь пары АБС, разделенных спейсерами с толщинами 3, 4 и 5 нм. $c-$ зависимости $C_{\text {e }}$ и $S_{\mathrm{h}}$ для структуры из трех АБС от толщины второго спейсера при толщине первого 3 нм (на вставке - схематическая энергетическая диаграмма такой структуры). Здесь АБС - 3 нм $\mathrm{Al}_{0.66} \mathrm{In}_{0.34} \mathrm{As}$, а спейсеры имеют зонную структуру, как у GaAs. (Цветной вариант рисунка представлен в электронной версии статьи). 


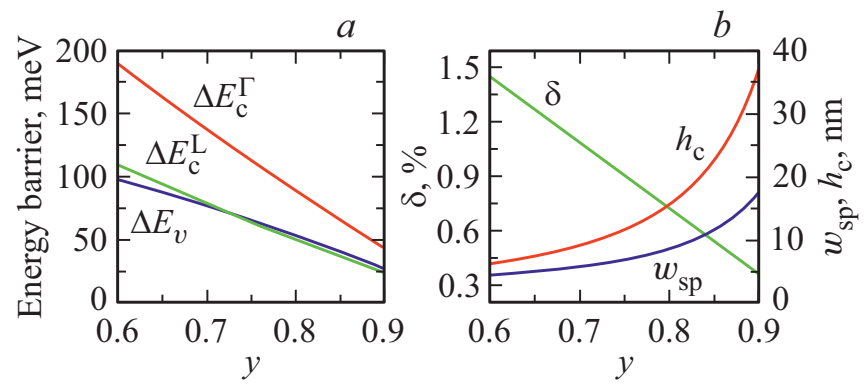

Рис. 6. Энергетические барьеры $\Delta E_{\mathrm{c}}^{\Gamma}$ и $\Delta E_{\mathrm{c}}^{\mathrm{L}}$ для электронов $\Gamma$ и L-долин соответственно, а также энергетический барьер $\Delta E_{v}$ для дырок, формируемые спейсером $\mathrm{GaAs}_{y} \mathrm{P}_{1-y}$ в $\mathrm{GaAs}(a)$. Рассогласование $\delta$, критическая толщина образования дислокаций $h_{\mathrm{c}}$ и толщина $w_{\mathrm{sp}}$, требуемая для компенсации напряжений 2.5 нм АБС $\mathrm{Al}_{0.66} \mathrm{In}_{0.34} \mathrm{As}$, для такого спейсера.

Наконец, рассмотрим структуру, предназначенную для блокирования электронов, состоящую из трех 2.5 нм АБС $\mathrm{Al}_{0.66} \mathrm{In}_{0.34} \mathrm{As}$, разделенных двумя спейсерами: $\mathrm{GaAs}_{y 1} \mathrm{P}_{1-y 1}$ и $\mathrm{GaAs}_{y 2} \mathrm{P}_{1-y 2}$. Параметры $y 1$ и $y 2$ варьировались нами в диапазоне от 0.6 до 0.9 для опре- деления оптимальных композиций спейсеров. На рис. 7 представлены степени подавления паразитного потока электронов $C_{\mathrm{e}}$ и полезного потока дырок $S_{\mathrm{h}}$, а также полная толщина структуры $w_{\text {tot }}$ и остаточный паразитный ток волноводной рекомбинации $j_{\text {res }}$ в модельном лазерном диоде для комбинации такой АБС-структуры и дырочного АБС hAB1 (GaInP) для различных параметров $y 1$ и $y 2$. Можно выбрать следующие оптимальные конструкции спейсеров: $9.1 \mathrm{Hм} \mathrm{GaAs}_{0.81} \mathrm{P}_{0.19}$ и $11.6 \mathrm{Hм}$ $\mathrm{GaAs}_{0.85} \mathrm{P}_{0.15}$. В этом случае получаются следующие значения параметров: $C_{\mathrm{e}}=2.3 \cdot 10^{5}, S_{\mathrm{h}}=90, w_{\mathrm{tot}}=27.9 \mathrm{Hм}$ и $j_{\text {res }}=1.0 \%$. Предложенная трехбарьерная структура (в табл. 2 и на рис. 7 обозначена как ТАВ) основана на простых тройных соединениях, не содержащих сурьму, и позволяет в 6.3 раза эффективнее подавлять волноводную рекомбинацию по сравнению с АБС еАВ1 (при использовании в комбинации с АБС hАB1).

\section{4. Заключение}

Таким образом, нами показано, что для GaAs-волновода могут быть подобраны конструкции АБС, позволяющие эффективно подавлять паразитную рекомбинацию
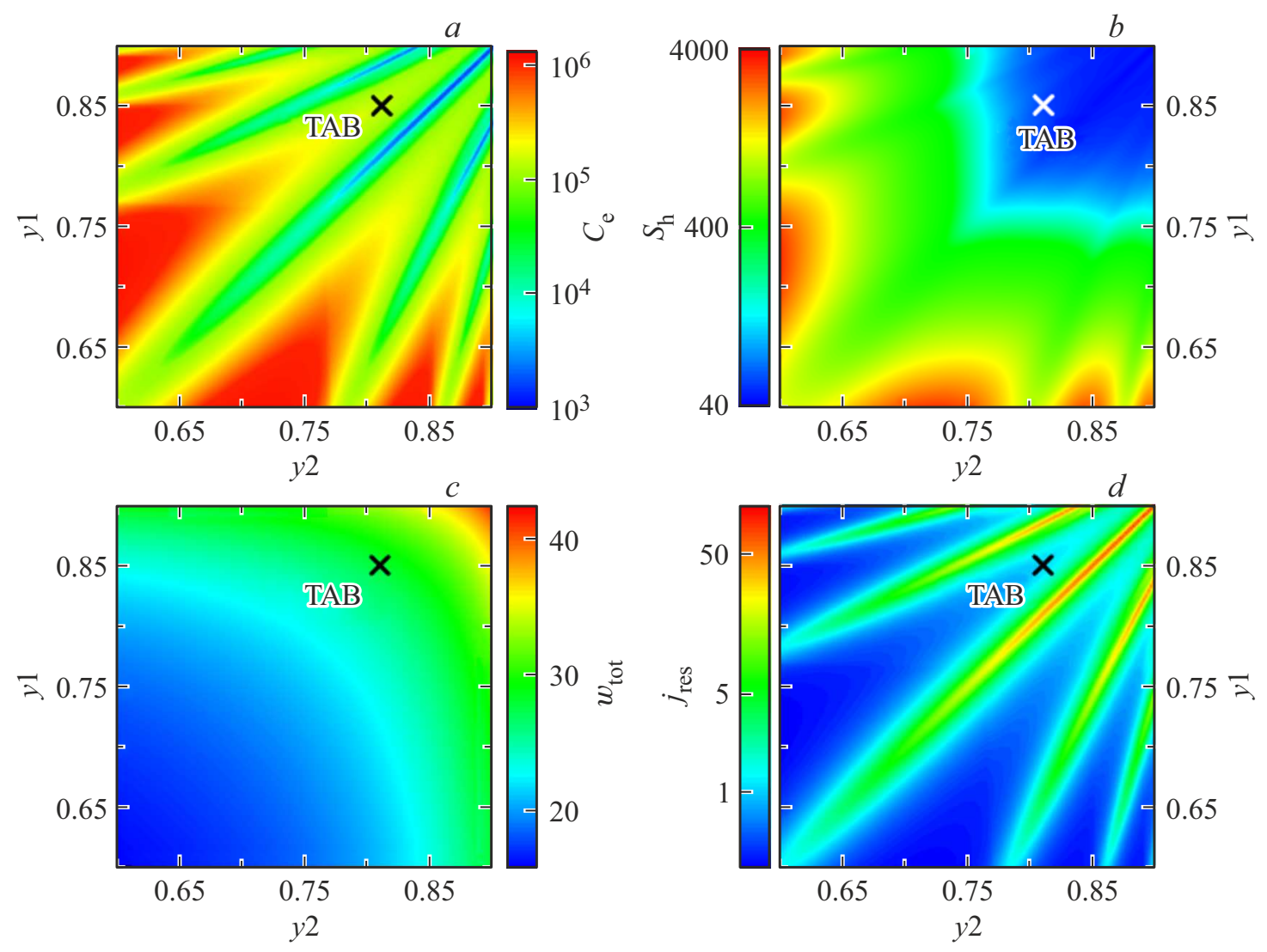

Рис. 7. Степени подавления паразитного потока электронов $C_{\mathrm{e}}(a)$ и полезного потока дырок $S_{\mathrm{h}}(b)$, а также полная толщина $w_{\text {tot }}\left(c\right.$, нм) АБС-структуры и остаточный паразитный ток волноводной рекомбинации $j_{\text {res }}\left(d, \mathrm{~A} / \mathrm{cm}^{2}\right)$ в модельном лазерном диоде для комбинации АБС-структуры и дырочного АБС hАВ1 для различных параметров $y 1$ и $y 2$. АБС-структура: три 2.5 нм АБС $\mathrm{Al}_{0.66} \mathrm{In}_{0.34} \mathrm{As}$, разделенные двумя спейсерами $\mathrm{GaAs}_{y 1} \mathrm{P}_{1-y 1}$ и $\mathrm{GaAs}_{y 2} \mathrm{P}_{1-y 2}$. (Цветной вариант рисунка представлен в электронной версии статьи). 
в мощных лазерных диодах с длиной волны генерации $\sim 1$ мкм. Оптимальными АБС для блокирования электронов являются $9.8 \mathrm{HM} \mathrm{Al}_{0.37} \mathrm{Ga}_{0.49} \mathrm{In}_{0.14} \mathrm{As}$ и $8.4 \mathrm{HM}$ $\mathrm{Al}_{0.4} \mathrm{Ga}_{0.6} \mathrm{As}_{0.86} \mathrm{Sb}_{0.14}$, снижающие паразитный поток носителей в $3.3 \cdot 10^{4}$ и $1.3 \cdot 10^{6}$ раз соответственно. Оптимальным АБС для блокирования дырок является $5.4 \mathrm{HM}$ $\mathrm{Ga}_{0.46} \mathrm{In}_{0.54} \mathrm{P}$ со степенью подавления паразитного транспорта, равной $1.0 \cdot 10^{5}$. Также для блокирования электронов предложена альтернативная АБС-конструкция на основе трех одинаковых барьеров $2.5 \mathrm{Hм} \mathrm{Al}_{0.66} \mathrm{In}_{0.34} \mathrm{As}$, разделенных спейсерами $9.1 \mathrm{Hм} \mathrm{GaAs}_{0.81} \mathrm{P}_{0.19}$ и $11.6 \mathrm{HM}$ $\mathrm{GaAs}_{0.85} \mathrm{P}_{0.15}$. Хотя данная структура не использует сурьму, в комбинации с дырочным АБС 5.4 нм $\mathrm{Ga}_{0.46} \operatorname{In}_{0.54} \mathrm{P}$ она позволяет эффективно снижать ток паразитной рекомбинации в GaAs волноводе (до 1\% от исходного тока в структуре без АБС). Преимуществом данной конструкции также является то, что она использует простые тройные соединения.

Следует отметить, что ослабление полезного потока в лазерной структуре (т.е. потока носителей, поступающих из волновода в активную область), по-видимому, приведет к замедлению динамики прибора (увеличению времени включения). Однако, поскольку мощные диодные лазеры, для которых использование АБС является наиболее актуальным, как правило, применяются в непрерывном или квазинепрерывном режиме генерации, такое замедление не должно быть критичным.

\section{Финансирование работы}

Исследование выполнено при финансовой поддержке Министерства образования и науки Российской Федерации (проект № 0791-2020-0002). Компьютерные расчеты выполнены в рамках Программы фундаментальных исследований НИУ ВШЭ. Л.В.А. благодарит U.S. Army Research Office, грант № W911NF-17-1-0432 за поддержку данной работы.

\section{Конфликт интересов}

Авторы заявляют, что у них нет конфликта интересов.

\section{Список литературы}

[1] H. Wenzel, P. Crump, A. Pietrzak, X. Wang, G. Erbert, G. Trankle. New J. Phys., 12, 085007 (2010).

[2] C. Frevert, P. Crump, F. Bugge, S. Knigge, G. Erbert. Semicond. Sci. Technol., 31, 025003 (2016).

[3] L.V. Asryan, S. Luryi. Solid-State Electron., 47, 205 (2003).

[4] A.E. Zhukov, N.V. Kryzhanovskaya, F.I. Zubov, Yu.M. Shernyakov, M.V. Maximov, E.S. Semenova, K. Yvind, L.V. Asryan. Appl. Phys. Lett. 100, 021107 (2012).

[5] F.I. Zubov, M.V. Maximov, Yu.M. Shernyakov, N.V. Kryzhanovskaya, E.S. Semenova, K. Yvind, L.V. Asryan, A.E. Zhukov. Electron. Lett., 51, 1106 (2015).

[6] L.V. Asryan, N.V. Kryzhanovskaya, M.V. Maximov, A.Yu. Egorov, A.E. Zhukov. Semicond. Sci. Technol., 26, 055025 (2011).
[7] L.V. Asryan, N.V. Kryzhanovskaya, M.V. Maximov, F.I. Zubov, A.E. Zhukov. J. Appl. Phys., 114, 143103 (2013).

[8] F.I. Zubov, M.E. Muretova, A.S. Payusov, M.V. Maximov, A.E. Zhukov, L.V. Asryan. Semiconductors, 54, 366 (2020).

[9] R. Tsu, L. Esaki. Appl. Phys. Lett., 22, 562 (1973).

[10] F.I. Zubov, M.E. Muretova, L.V. Asryan, E.S. Semenova, M.V. Maximov, A.E. Zhukov. J. Appl. Phys., 124, 133105 (2018).

[11] I. Vurgaftman, J.R. Meyer, L.R. Ram-Mohan. J. Appl. Phys., 89, 5815 (2001).

[12] S. Adachi. Properties of Semiconductor Alloys: Group-IV, III-V and II-VI Semiconductors (Wiley, 2009).

\section{Design of blocking layers for suppression of parasitic recombination in high-power laser diodes with GaAs waveguide}

\author{
M.E. Muretova ${ }^{1}$, F.I. Zubov ${ }^{\mathbf{1}, 2}$, L.V. Asryan ${ }^{3}$, \\ Yu.M. Shernyakov ${ }^{4}$, M.V. Maximov ${ }^{1,2}$, A.E. Zhukov ${ }^{2,1}$ \\ ${ }^{1}$ Alferov University, \\ 194021 St. Petersburg, Russia \\ ${ }^{2}$ National Research University \\ Higher School of Economics, \\ 190008 St. Petersburg, Russia \\ ${ }^{3}$ Virginia Polytechnic Institute and State University, \\ Blacksburg, Virginia 24061, USA \\ ${ }^{4}$ loffe Institute, \\ 194021 St. Petersburg, Russia
}

\begin{abstract}
Using numerical simulation, a search is carried out for designs of asymmetric barrier layers (ABLs) for a laser diode having GaAs waveguide and emitting at the wavelength $\lambda=980 \mathrm{~nm}$. A pair of ABLs, adjoining the active region on both sides, blocks undesired charge carrier flows and suppresses parasitic spontaneous recombination in the waveguide layers. Optimal designs of ABLs based on $\mathrm{AlGaAsSb}$ and $\mathrm{GaInP}$ for blocking electrons and holes, respectively, are proposed that make it possible to reduce the parasitic recombination current down to less than $1 \%$ of the initial value. To suppress electron transport, an alternative structure based on three identical AlInAs barriers is also proposed. The GaAsP spacers separating these barriers from each other have different thicknesses. Due to this, its own set of quasi-bound (resonant) states is formed in each spacer that is different from the neighbor spacer set of states. As a result of this, the resonant tunneling channels are blocked: the parasitic electron flow is reduced by several tens of times in comparison with the case of spacers of equal thickness.
\end{abstract}

